\title{
Cobalt-catalyzed oxidation of terpenes: Co-MCM-41 as an efficient shape-selective heterogeneous catalyst for aerobic oxidation of isolongifolene under solvent-free conditions
}

\author{
Patricia A. Robles-Dutenhefner ${ }^{a}$, Kelly A. da Silva Rocha ${ }^{b}$, Edésia M.B. Sousa ${ }^{c}$, Elena V. Gusevskaya ${ }^{b, *}$ \\ ${ }^{a}$ Departamento de Qúmica, Universidade Federal de Ouro Preto, 35400-000 Ouro Preto, MG, Brazil \\ ${ }^{\mathrm{b}}$ Departamento de Química, Universidade Federal de Minas Gerais, 31270-901 Belo Horizonte, MG, Brazil

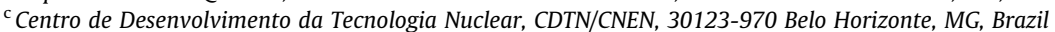

\section{A R T I C L E I N F O}

\section{Article history:}

Received 23 February 2009

Revised 7 April 2009

Accepted 19 April 2009

Available online 15 May 2009

\section{Keywords:}

Isolongifolene

Autoxidation

Cobalt catalysts

MCM-41

\begin{abstract}
A B S T R A C T
Cobalt-containing MCM-41 was used as a heterogeneous catalyst for the liquid-phase aerobic oxidation of isolongifolene, one of the most available sesquiterpenes. The material was prepared by direct incorporation of cobalt into the framework through the hydrothermal method, and was characterized by ICPAES, $\mathrm{N}_{2}$ adsorption-desorption, TEM, XRD, SAXS, XPS, and $\mathrm{H}_{2}$-TPR techniques. Characterizations suggest that cobalt introduced into MCM-41 exists mainly in a single-site $\mathrm{Co}^{2+}$ state, whereas the amounts of the extraframework $\mathrm{CO}_{3} \mathrm{O}_{4}$ are relatively low. The oxidation of isolongifolene under mild solvent-free conditions results mainly (90\% selectivity) in isolongifolen-9-one, a compound which occupies a vintage place in modern perfume industry. The total concentration of oxygenated products in the final mixtures reaches $90 \mathrm{wt} \%$. The catalyst undergoes no metal leaching, and can be easily recovered and re-used. A silica-included cobalt catalyst prepared through a conventional sol-gel method shows a catalytic activity comparable with that of Co-MCM-41; however, selectivity is much lower.
\end{abstract}

(c) 2009 Elsevier Inc. All rights reserved.

\section{Introduction}

The development of catalytic aerobic oxidations of organic compounds to replace conventional stoichiometric processes in fine chemical industry represents a constant goal in catalysis research [1-3]. In this field, particularly important for green chemistry, the great challenges are the introduction of solvent-free technologies to minimize the amounts of effluents and the use of solid materials in liquid-phase reactions to allow facile catalyst recovering.

A conventional heterogenization of homogeneous redox systems on a solid support by impregnation often faces serious problems in liquid-phase reactions due to a rapid leaching of metal ions. On the other hand, the application of heterogeneous gasphase processes in fine chemistry is restricted by limited volatility and thermal stability of complex substrates. Among the most promising approaches to the development of truly heterogeneous catalysts for liquid-phase oxidations is the inclusion of redox-active metals in a solid material during the synthesis of the solid, i.e., isomorphic substitution $[1,4]$. Such site-isolation of active metal ions in the framework positions of solid matrices often not only results in higher stability of these catalysts toward leaching but also prevents metal aggregation to less reactive species [1].

\footnotetext{
* Corresponding author. Fax: +55 3134095700.

E-mail address: elena@ufmg.br (E.V. Gusevskaya).
}

Ordered mesoporous molecular sieves, M41S, discovered by Mobil Company in 1992, can be synthesized from a solubilized silica or silica/aluminum source in the presence of the surfactant, which acts as a structure template directing the formation of solids from silicate/aluminosilicate gels [5,6]. These materials possess regular arrays of large uniform channels and high surface areas (above $700 \mathrm{~m}^{2}$ ). MCM-41, the most important member of this family, exhibits a hexagonal arrangement of mesopores, whose dimensions $(2-10 \mathrm{~nm})$ can be controlled through the choice of surfactant, auxiliary chemicals, and synthesis conditions. These mesoporous materials are very attractive for the development of new heterogeneous catalysts for the fine chemical industry, which especially needs large pore catalysts for the conversion of bulky molecules. Many efforts have been devoted to the synthesis of transition metal-containing MCM-41 (the so-called redox molecular sieves or metallosilicates) and its application in liquid-phase oxidations of hydrocarbons [4,7]. In particular, several groups have reported the preparation of cobalt-containing MCM- 41 by the modification of molecular sieves via both post-synthesis impregnation $[7,8]$ and inclusion of the metal during the synthesis of the molecular sieve [9-12].

The aim of the present work was to apply MCM-41-included cobalt catalysts for the liquid-phase aerobic oxidation of isolongifolene. Isolongifolene is a tricyclic sesquiterpene which can be easily obtained by acid-catalyzed isomerization of longifolene 
[13-15], one of the most abundant sesquiterpenes found in relatively large amounts in Indian turpentine oil. Oxygenated derivatives of isolongifolene occupy a vintage place in modern perfume industry because of their extremely rich woody and floral odor [16-19]. Most of the reported processes for the oxidation of isolongifolene are non-catalytic reactions employing traditional oxidants in stoichiometric amounts (permanganates, dichromates, peroxo compounds, etc.) [16-22]. These reactions usually result in the complex mixtures of oxygenated products.

Terpenic compounds, in general, are an important renewable feedstock for flavor and fragrance industries. For several years, we have been interested in catalytic transformations of terpenes to value-added chemicals, in particular, via cobalt [23-26] and palladium [27-30] catalyzed oxidations. The most abundant nonfunctionalized monoterpenes, such as limonene and pinenes, have been mainly used in our previous works.

In the present work, we report a simple and efficient aerobic oxidation of isolongifolene into a conjugated ketone, which is highly important for perfume industry and can be obtained in excellent yields under mild solvent-free conditions. A Co-MCM41 material, which contains cobalt incorporated into the framework, is used as a heterogeneous, low cost, and easily recyclable catalyst. The performance of Co-MCM-41 is compared with that of a silica-included cobalt material prepared by a conventional sol-gel method without the surfactant.

\section{Experimental}

All reagents were purchased from commercial sources and were used as received. Isolongifolene was kindly donated by Professor J.C. Bayón (Universidad Autónoma de Barcelona).

\subsection{Catalyst preparation and characterization}

The $5.0 \mathrm{wt} \% \mathrm{Co} / \mathrm{SiO}_{2}$ catalyst (denoted as $\mathrm{Co}-\mathrm{SiO}_{2} /$ sol-gel) was prepared by a sol-gel method using tetraethoxysilane $(0.9 \mathrm{~g}$, TEOS, Sigma-Aldrich) and $\mathrm{CoCl}_{2} \times 6 \mathrm{H}_{2} \mathrm{O}$ (15.2 g, Sigma-Aldrich) as precursors. The sol was obtained from a TEOS/ethanol/water mixture in a $1 / 3 / 10$ molar ratio with the addition of $\mathrm{HCl}$ and $\mathrm{HF}$ (up to $\mathrm{pH}$ 2.0 ) as catalysts. The sample was prepared in a monolithic shape, dried at $110^{\circ} \mathrm{C}$ for $48 \mathrm{~h}$, and thermally treated for $2 \mathrm{~h}$ at $900{ }^{\circ} \mathrm{C}$ in air.

The catalyst $3.3 \mathrm{wt} \% \mathrm{Co} / \mathrm{MCM}-41$ catalyst (denoted as Co-MCM41) was prepared by the direct incorporation of Co into the MCM-41 framework aiming for the isomorphous substitution of Si by Co ions. TEOS and $\mathrm{CoCl}_{2} \times 6 \mathrm{H}_{2} \mathrm{O}$ (Sigma-Aldrich) were used as precursors, and hexadecyltrimethylammonium bromide (C16-TAB, Sigma-Aldrich) was used as a structure template. The C16-TAB solution in water was added to the solution of TEOS $(2.5 \mathrm{~g})$ in aqueous tetramethylammonium hydroxide (TMAOH, $25 \mathrm{wt} \%$, Sigma-Aldrich). The mixture was stirred for 30 min before $\mathrm{CoCl}_{2}(1.2 \mathrm{~g})$ and remaining TEOS $(21.0 \mathrm{~g})$ were added. After additional mixing at $40{ }^{\circ} \mathrm{C}$ for $24 \mathrm{~h}$, the mixture was placed in an autoclave at $100^{\circ} \mathrm{C}$ for $24 \mathrm{~h}$ and was then cooled to room temperature. The resulting solid was recovered by filtration, washed first with de-ionized water and then with ethanol, and dried at $40^{\circ} \mathrm{C}$. The pre-dried solid was heated from room temperature to $550^{\circ} \mathrm{C}$ under flowing nitrogen and was calcinated for $3 \mathrm{~h}$ at $550{ }^{\circ} \mathrm{C}$ under flowing air to remove the residual organics. The TEOS/C16-TAB/TMAOH/water molar ratio was $1.0 /$ $0.12 / 0.28 / 26.2$.

The determination of total Co contents was done by inductively coupled plasma atomic emission spectrometry (ICP-AES) on a Spectro Ciros CCD instrument.

The textural characteristics of the catalysts were determined from nitrogen adsorption isotherms (Autosorb-Quantachrome
NOVA-1200 instrument, nitrogen, $-196{ }^{\circ} \mathrm{C}$ ). Samples were outgassed for $2 \mathrm{~h}$ at $300{ }^{\circ} \mathrm{C}$ before analysis. Specific surface areas and average pore diameter were determined by the Brunauer-Emmett-Teller (BET) equation. The pore size distributions were calculated from the desorption isotherms using the Barrett-Joyner-Halenda (BJH) method.

Transmission electron microscopy (TEM) images were obtained using a JEOL-JEM 1200 instrument operating at an accelerating voltage of $120 \mathrm{kV}$.

Powder X-ray diffractometry (XRD) measurements were performed on a Rigaku model Geigerflex-3034 equipment using a $\mathrm{Co}(\mathrm{K} \alpha)$ radiation scanning from 4 to $70^{\circ}(2 \theta)$ to register the presence of cobalt oxide phases. Samples were dried previously and pulverized.

Small-angle X-ray scattering (SAXS) experiment at the D11ASAXS beam line was performed at the LNLS synchrotron laboratory, Campinas, Brazil. The SAXS setup was equipped with a Si(111) monochromator, giving a horizontally focused X-ray beam. The incident X-ray wavelength $\lambda$ was $1.5494 \AA$, and the scattering angle $2 \theta$ was approximately $0-10^{\circ}$.

X-ray photoelectron spectroscopy (XPS) measurements were performed on a VG-Microtech Multilab 3000 spectrometer equipped with a hemispherical electron analyzer and a $\operatorname{Mg}(\mathrm{K} \alpha)$ $\left(h v=1253.6 \mathrm{eV}, 1 \mathrm{eV}=1.6302 \times 10^{-19} \mathrm{~J}\right) 300 \mathrm{~W}$ X-ray source. The spectra were collected at a pass energy of $50 \mathrm{eV}$.

Temperature-programmed reduction with hydrogen $\left(\mathrm{H}_{2}-\mathrm{TPR}\right)$ was performed on a Quantachrome-ChemBET 300 instrument equipped with a thermal conductivity detector. The experiments were performed between 30 and $900^{\circ} \mathrm{C}\left(10^{\circ} \mathrm{C} / \mathrm{min}\right)$ in a flow of a $\mathrm{N}_{2} / \mathrm{H}_{2}$ mixture containing $5 \mathrm{vol} \%$ of $\mathrm{H}_{2}$.

\subsection{Catalytic oxidation experiments}

Reactions were carried out in a glass reactor equipped with a magnetic stirrer and a sampling system and connected to a gas burette to monitor the oxygen uptake. In a typical run, a mixture of isolongifolene $(14 \mathrm{mmol})$, dodecane $(4.4 \mathrm{mmol}$, internal standard), and the catalyst (0.1-0.2 g, ca. 2.8-5.6 wt\%) was intensively stirred at $80^{\circ} \mathrm{C}$ and at an oxygen pressure of $1 \mathrm{~atm}$ for the reported time. The reactions were followed by measuring the uptake of oxygen and by gas chromatography (GC) using dodecane as an internal standard (Shimadzu 17 instrument, Carbowax $20 \mathrm{M}$ capillary column, temperature program: $80^{\circ} \mathrm{C}$, isothermal, $3 \mathrm{~min} ; 10^{\circ} \mathrm{C} / \mathrm{min}$ up to $220^{\circ} \mathrm{C}$; and $220^{\circ} \mathrm{C}$, isothermal, $5 \mathrm{~min}$ ). At appropriate time intervals, stirring was stopped and after the quick settling of the catalyst aliquots were taken, diluted 20 -fold with cyclohexane, and were analyzed by GC.

Catalyst recycling experiments were performed as follows: after the reaction, the catalyst was centrifuged, washed with cyclohexane, and reused. To control metal leaching, the catalyst was removed at the reaction temperature after $2 \mathrm{~h}$ and the solution was allowed to react further.

Products 2-5 were isolated by column chromatography (silica) using mixtures of hexane and $\mathrm{CH}_{2} \mathrm{Cl}_{2}$ as eluents, and were identified by GC/MS (Shimadzu QP2010-PLUS instrument, $70 \mathrm{eV}$ ) and NMR (Bruker DRX-400 instrument, tetramethylsilane, $\mathrm{CDCl}_{3}$ ).

Isolongifolen-9-one (2): MS (m/z/rel.int.): 218/43 ( $\left.{ }^{+}\right) ; 203 / 10$ $\left(\mathrm{M}^{+}-\mathrm{CH}_{3}\right) ; 176 / 40 ; 175 / 90 ; 162 / 67 ; 147 / 60 ; 133 / 23 ; 119 / 30$; $105 / 33 ; 91 / 42 ; 77 / 23 ; 55 / 28 ; 43 / 23 ; 41 / 100 .{ }^{1} \mathrm{H}$ NMR, $\delta_{\mathrm{H}}(\mathrm{J}, \mathrm{Hz})$ : 0.99 (s, 3H, $\left.\mathrm{C}^{14} \mathrm{H}_{3}\right) ; 1.05$ (s, 3H, $\left.\mathrm{C}^{12} \mathrm{H}_{3}\right) ; 1.09$ (s, 3H, $\left.\mathrm{C}^{15} \mathrm{H}_{3}\right) ; 1.14$ (s, $\left.3 \mathrm{H}, \mathrm{C}^{13} \mathrm{H}_{3}\right) ; 1.29-1.33\left(\mathrm{~m}, 1 \mathrm{H}, \mathrm{C}^{5} \mathrm{HH}\right) ; 1.41\left(\mathrm{~d}, 1 \mathrm{H}, \mathrm{C}^{11} \mathrm{HH}\right.$, $\left.{ }^{2} J=10.0\right) ; 1.57-1.65\left(\mathrm{~m}, 1 \mathrm{H}, \mathrm{C}^{4} \mathrm{HH}\right) ; 1.68\left(\mathrm{dd},{ }^{2} J=10.0,{ }^{3} J=2.0\right.$, $\mathrm{C}^{11} \mathrm{HH}$ ); $1.73-1.77$ (m, 1H, C $\left.\mathrm{C}^{4} \mathrm{HH}\right) ; 1.91-1.98\left(\mathrm{~m}, 2 \mathrm{H}, \mathrm{C}^{3} \mathrm{H}, \mathrm{C}^{5} \mathrm{HH}\right)$; $2.07\left(\mathrm{~d}, 1 \mathrm{H}, \mathrm{C}^{8} \mathrm{HH},{ }^{2} \mathrm{~J}=16.0\right) ; 2.38\left(\mathrm{~d}, 1 \mathrm{H}, \mathrm{C}^{8} \mathrm{HH},{ }^{2} \mathrm{~J}=16.0\right) ; 5,70(\mathrm{~s}$, $\left.1 \mathrm{H} \mathrm{C}{ }^{10} H\right) .{ }^{13} \mathrm{C}$ NMR, $\delta_{\mathrm{C}}: 24.33\left(C^{4}\right) ; 24.57\left(C^{13}\right) ; 25.40\left(C^{14}\right) ; 25.76$ $\left(C^{12}\right)$; $26.97\left(C^{15}\right) ; 27.83\left(C^{5}\right) ; 34.45\left(C^{7}\right) ; 36.70\left(C^{11}\right) ; 44.12\left(C^{2}\right)$; 
$46.50\left(C^{3}\right) ; 49.85\left(C^{8}\right), 58.64\left(C^{6}\right) ; 116.81\left(C^{10}\right) ; 184.17\left(C^{1}\right) ; 200.38$

$\left(C^{9}\right)$. The data are consistent with those reported in $[19,31]$.

Isolongifolen-9-ol (3): MS (m/z/rel.int.): 220/10 ( $\left.\mathrm{M}^{+}\right) ; 205 / 10$ $\left(\mathrm{M}^{+}-\mathrm{CH}_{3}\right) ; 164 / 23 ; 177 / 20 ; 137 / 100 ; 121 / 35 ; 107 / 21 ; 91 / 20 ; 41 /$ 25. ${ }^{1} \mathrm{H}$ NMR, $\delta_{\mathrm{H}}(\mathrm{J}, \mathrm{Hz}): 0.97\left(\mathrm{~s}, 3 \mathrm{H}, \mathrm{C}^{12} \mathrm{H}_{3}\right) ; 1.05\left(\mathrm{~s}, 3 \mathrm{H}, \mathrm{C}^{12} \mathrm{H}_{3}\right)$; $1.07\left(\mathrm{~s}, 3 \mathrm{H}, \mathrm{C}^{15} \mathrm{H}_{3}\right) ; 1.14\left(\mathrm{~s}, 3 \mathrm{H}, \mathrm{C}^{13} \mathrm{H}_{3}\right) ; 1.29-1.33\left(\mathrm{~m}, 2 \mathrm{H}, \mathrm{C}^{8} \mathrm{HH}\right.$, $\left.\mathrm{C}^{5} \mathrm{HH}\right) ; 1.41$ (d, $\left.1 \mathrm{H}, \mathrm{C}^{11} \mathrm{HH},{ }^{2} \mathrm{~J}=10.0\right) ; 1.48-1.52\left(\mathrm{~m}, 1 \mathrm{H}, \mathrm{C}^{8} \mathrm{HH}\right)$; $1.57-1.65\left(\mathrm{~m}, 1 \mathrm{H}, \mathrm{C}^{4} \mathrm{HH}\right) ; 1.68$ (dd, $\left.{ }^{2} J=10.0,{ }^{3} \mathrm{~J}=2.0, \mathrm{C}^{11} \mathrm{H} H\right)$; 1.73-1.77 (m, $\left.1 \mathrm{H}, \mathrm{C}^{4} \mathrm{HH}\right)$; $1.78-1.85\left(\mathrm{~m}, 1 \mathrm{H}, \mathrm{C}^{3} \mathrm{H}\right) ; 1.91-1.98(\mathrm{~m}$, $\left.1 \mathrm{H}, \mathrm{C}^{5} \mathrm{HH}\right) ; 4.59\left(\mathrm{dd},{ }^{3} J=9.5,{ }^{3} J=4.0, \mathrm{C}^{9} \mathrm{H}\right) ; 5,33\left(\mathrm{~d}, 1 \mathrm{H},{ }^{3} J=4.0\right.$, $\left.\mathrm{C}^{10} \mathrm{H}\right) .{ }^{13} \mathrm{C}$ NMR, $\delta_{\mathrm{C}}: 24.63\left(C^{4}\right) ; 24.97\left(C^{13}\right) ; 25.21\left(C^{12}\right) ; 26.66$ $\left(C^{14}\right)$; $28.63\left(C^{5}\right)$; $28.74\left(C^{15}\right)$; $33.08\left(C^{7}\right)$; $36.59\left(C^{8}\right)$, $38.55\left(C^{11}\right)$; $42.28\left(C^{2}\right) ; 46.79\left(C^{3}\right) ; 56.80\left(C^{6}\right) ; 81.21\left(C^{9}\right) ; 109.95\left(C^{10}\right) ; 161.27$ $\left(C^{1}\right)$. The data are consistent with those reported in [19].

Isolongifolene epoxide (4): MS ( $m / z /$ rel.int.): 220/60 $\left(\mathrm{M}^{+}\right) ; 206 /$ 10; 205/45 $\left(\mathrm{M}^{+}-\mathrm{CH}_{3}\right) ; 191 / 100 ; 177 / 50 ; 164 / 75 ; 149 / 83 ; 135 / 34$; $121 / 94 ; 107 / 63 ; 105 / 28 ; 93 / 43 ; 83 / 47 ; 67 / 25 ; 65 / 22 ; 55 / 48 .{ }^{13} \mathrm{C}$ NMR, $\delta_{\mathrm{C}}: 20.80,25.28,26.63,27.25\left(\mathrm{CH}_{3}\right) ; 22.04,24.44,24.96$, 32.71, $36.73\left(\mathrm{CH}_{2}\right)$; 46.62, $57.05(\mathrm{CH}) ; 30.16,39.26,52.32,72.55$

(C). The data are consistent with those reported in $[17,18]$.

Isolongifolan-10-one (5): MS (m/z/rel.int.): 220/22 ( $\left.{ }^{+}\right) ; 205 / 15$ $\left(\mathrm{M}^{+}-\mathrm{CH}_{3}\right) ; 191 / 26 ; 164 / 23 ; 149 / 25 ; 121 / 34 ; 105 / 27 ; 92 / 20 ; 90 /$ $22 ; 83 / 28 ; 79 / 25 ; 65 / 20 ; 67 / 21 ; 55 / 60 ; 43 / 46 ; 41 / 100 .{ }^{13} \mathrm{C}$ NMR, $\delta_{\mathrm{C}}: 23.16,23.40,25.28,32.70\left(\mathrm{CH}_{3}\right) ; 23.42,26.43,36.88 ; 37.86$, $40.09\left(\mathrm{CH}_{2}\right) ; 49.04,60.61(\mathrm{CH}) ; 33.52,38.32,60.00,211.34(\mathrm{C})$. The data are consistent with those reported in $[17,18]$.

\section{Results and discussion}

\subsection{Characterization of the catalysts}

The results of the elemental analysis of the prepared samples, their BET surface areas, average pore sizes, and pore volumes are presented in Table 1. Nitrogen adsorption-desorption isotherms and corresponding BJH pore size distributions are shown in Figs. $1-4$. As it can be seen, the Co-MCM-41 catalyst shows a much higher specific surface area $\left(781 \mathrm{~m}^{2} \mathrm{~g}^{-1}\right)$ than $\mathrm{Co}-\mathrm{SiO}_{2} / \mathrm{sol}$-gel $\left(187 \mathrm{~m}^{2} \mathrm{~g}^{-1}\right)$, and a much smaller average BET pore diameter ( $3.4 \mathrm{~nm}$ for $\mathrm{Co}-\mathrm{MCM}-41$ vs. $22.9 \mathrm{~nm}$ for $\mathrm{Co}-\mathrm{SiO}_{2} /$ sol-gel).

The Co-MCM-41 sample exhibits a type IV isotherm (in the UPAC classification) (Fig. 1) with a sharp inflection step at a relative pressure range from 0.2 to 0.4 , characteristic of capillary condensation in uniform mesopores [32,33], which suggests that the Co-MCM-41 sample was successfully synthesized. The isotherm at $P / P_{0}<0.2$ represents a monolayer adsorption on the walls of mesopores, whereas that at $P / P_{0}>0.4$ represents a multilayer adsorption on the external surface of the particles. The isotherm shows two well-defined hysteresis loops. The one corresponding to the capillary condensation/evaporation (at $P / P_{\mathrm{o}}=0.2-0.4$ ) exhibits the $\mathrm{H} 1$ type in the UPAC classification and has almost parallel steep adsorption and desorption branches, which is characteristic of the mesoporous materials with cylindrical pore geometry, high degree of pore size uniformity, and facile pore connectivity [34]. The second hysteresis loop with parallel and almost horizontal branches at relative pressures close to the saturated vapor pressure exhibits the $\mathrm{H} 4$ type. The appearance of the $\mathrm{H} 4$ hysteresis loops in the MCM-41 materials was suggested to be related to the presence

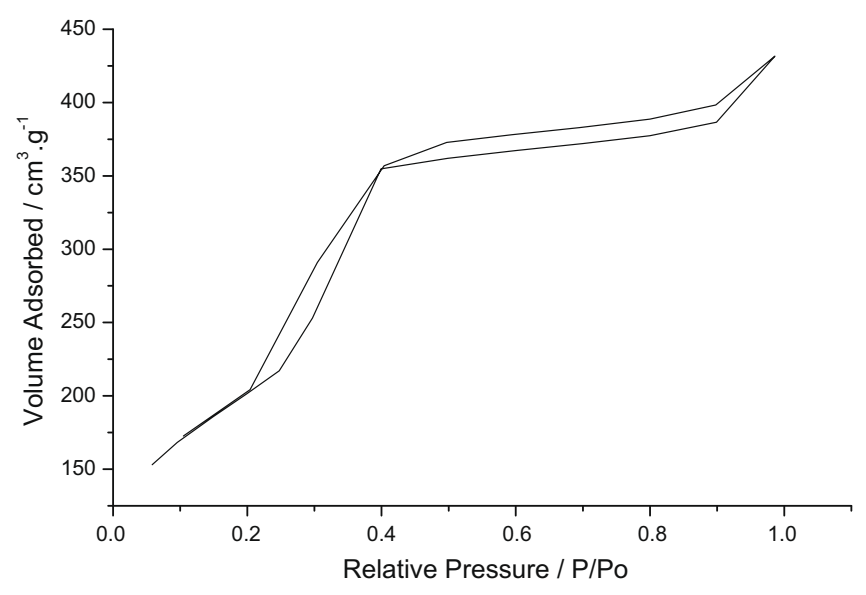

Fig. 1. Nitrogen adsorption-desorption isotherm for the Co-MCM-41 sample at $-196^{\circ} \mathrm{C}$.

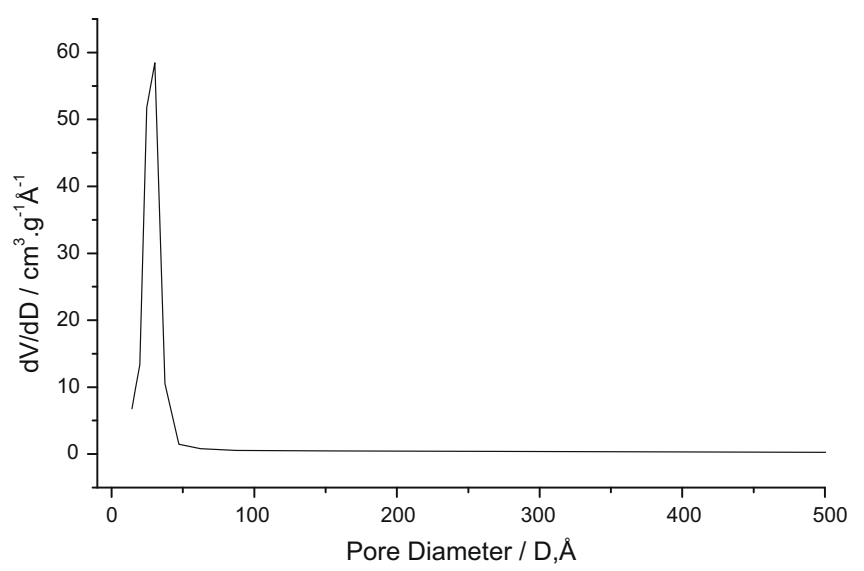

Fig. 2. Pore size distribution for the Co-MCM-41 sample calculated from the desorption isotherm using the BJH method.

of large mesopores embedded in a matrix with pores of much smaller size [34]. The pore size distribution in our Co-MCM-41 sample is quite narrow and monomodal, showing a peak pore diameter at $2.4 \mathrm{~nm}$ with a peak width of ca. $1.0 \mathrm{~nm}$ at half-maximum (Fig. 2).

Thus, the high BET surface area, nitrogen adsorption isotherm, and narrow pore size distribution confirm the uniform mesoporosity and organized structure of the Co-MCM-41 sample. The ordered hexagonal structure of Co-MCM-41 was further confirmed by TEM (Fig. 5). Although the micrograph does not allow to determine the shape of the individual pores, a regularity of pore sizes can be seen unambiguously.

The isotherm for the $\mathrm{Co}-\mathrm{SiO}_{2} /$ sol-gel material also corresponds to the type IV (Fig. 3). The appearance of the hysteresis loop of type H1 may indicate, in this case, that the mesoporous material consists of agglomerates of approximately spherical particles of fairly uniform size and array [32]. The pore size distribution in the

Table 1

Elemental analysis data and textural properties of $\mathrm{Co}-\mathrm{MCM}-41$ and $\mathrm{Co}-\mathrm{SiO}_{2} / \mathrm{sol}_{\text {-gel. }}$

\begin{tabular}{|c|c|c|c|c|c|}
\hline \multirow[t]{2}{*}{ Sample } & \multirow[t]{2}{*}{ Co content (wt\%) } & \multirow[t]{2}{*}{ BET surface area $\left(\mathrm{m}^{2} \mathrm{~g}^{-1}\right)$} & \multirow[t]{2}{*}{ Total pore volume $\left(\mathrm{cm}^{3} \mathrm{~g}^{-1}\right)$} & \multicolumn{2}{|c|}{ Average pore diameter } \\
\hline & & & & $\mathrm{BET}(\mathrm{nm})$ & $\mathrm{BJH}(\mathrm{nm})$ \\
\hline Co-MCM-41 & 3.3 & 781 & 0.67 & 3.4 & 2.4 \\
\hline $\mathrm{Co}-\mathrm{SiO}_{2} /$ sol-gel & 5.0 & 187 & 1.06 & 22.9 & 14.8 \\
\hline
\end{tabular}




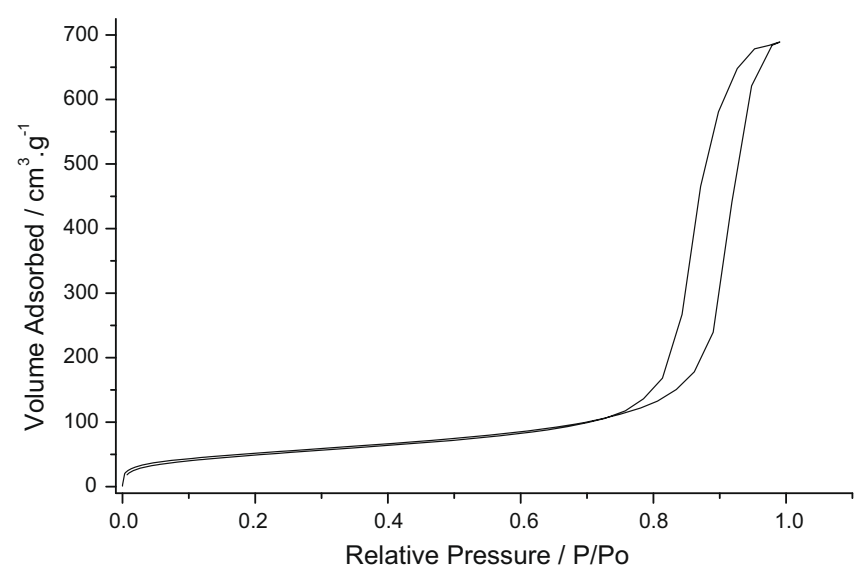

Fig. 3. Nitrogen adsorption-desorption isotherm for the $\mathrm{Co}-\mathrm{SiO}_{2} /$ sol-gel sample at $-196^{\circ} \mathrm{C}$.

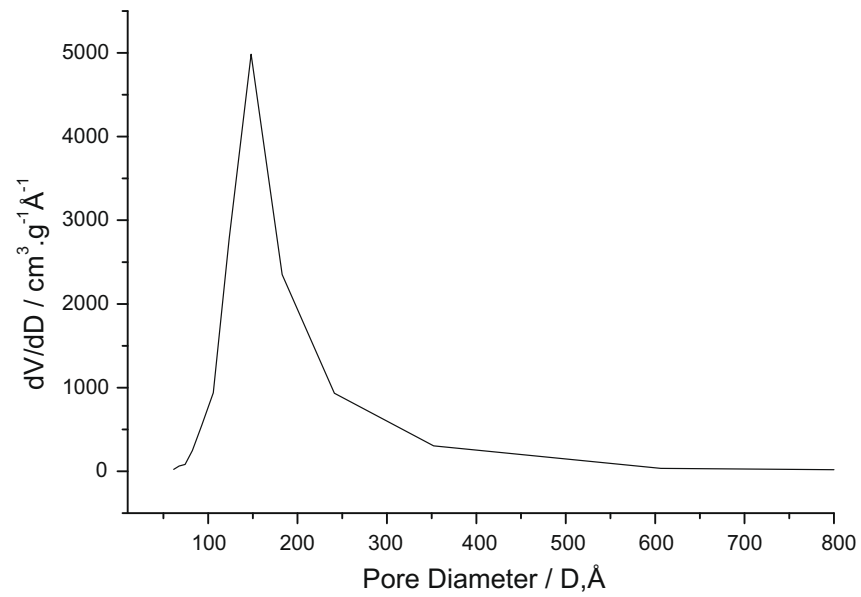

Fig. 4. Pore size distribution for the $\mathrm{Co}-\mathrm{SiO}_{2} /$ sol-gel sample calculated from the desorption isotherm using the $\mathrm{BJH}$ method.

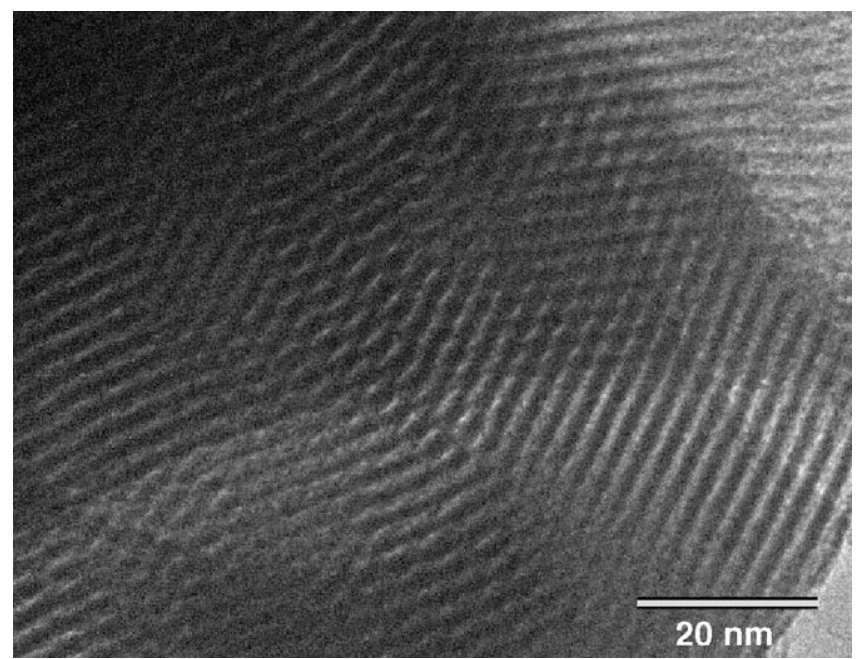

Fig. 5. HR-TEM micrograph of the Co-MCM-41 sample.

$\mathrm{Co}-\mathrm{SiO}_{2} /$ sol-gel sample is also monomodal, showing a peak pore diameter at $14.8 \mathrm{~nm}$, and quite narrow, albeit much broader than that of Co-MCM-41 as the peak width at half-maximum is ca. $6.5 \mathrm{~nm}$ (Fig. 4).

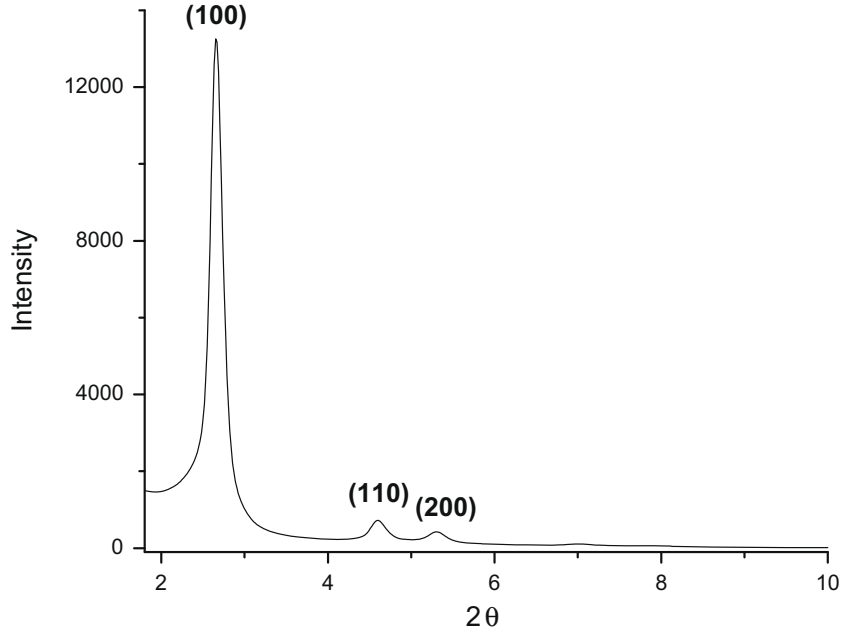

Fig. 6. SAXS pattern for the Co-MCM-41 sample.

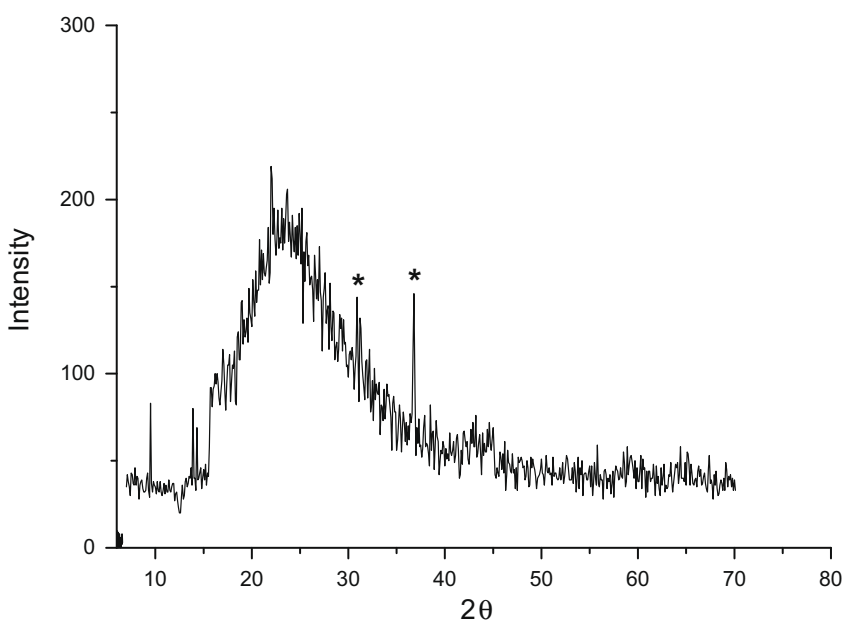

Fig. 7. XRD pattern for the Co-MCM-41 sample. ${ }^{*} \mathrm{Co}_{3} \mathrm{O}_{4}$.

The structure of most ordered mesoporous materials shows a long range ordering of amorphous elements which can be studied by a SAXS technique. The synchrotron radiation small-angle pattern for the Co-MCM-41 (Fig. 6) exhibits well-resolved (100), (110), and (200) reflections at $2 \theta=2.66^{\circ}, 4.57^{\circ}$, and $2.23^{\circ}$, respectively, indicating the formation of a well-ordered structure with a hexagonal pore array [6]. The peaks can be indexed to a hexagonal lattice structure with a $d_{100}$ spacing of $3.34 \mathrm{~nm}$ and a unit cell parameter $\left(a=2 d_{100} / \sqrt{ } 3\right)$ of $3.85 \mathrm{~nm}$. Thus, the SAXS experiment has also confirmed that the prepared cobalt-containing material has a typical structure of MCM-41 silicates, which are amorphous, however, diffract X-rays by pore walls of the repeating regular structure.

The higher angle XRD pattern for Co-MCM-41 (Fig. 7) shows the presence of weak peaks characteristic of a $\mathrm{CO}_{3} \mathrm{O}_{4}$ phase indicating the formation of small amounts of cobalt oxide microcrystallites on the walls of the metallosilicate. The diffractogram of the $\mathrm{Co}-\mathrm{SiO}_{2} /$ sol-gel sample exhibits more intense peaks of the $\mathrm{Co}_{3} \mathrm{O}_{4}$ phase, whereas the matrix is amorphous (Fig. 8).

The surface composition of the catalysts and the chemical states of surface cobalt species were investigated by X-ray photoelectron spectroscopy. The Co $2 \mathrm{p}$ photoelectron spectra of the as-synthesized $\mathrm{Co}-\mathrm{SiO}_{2} /$ sol-gel and $\mathrm{Co}-\mathrm{MCM}-41$ samples are presented in Fig. 9. The high binding energy of the main spin-orbit Co $2 \mathrm{p}_{3 / 2}$ 


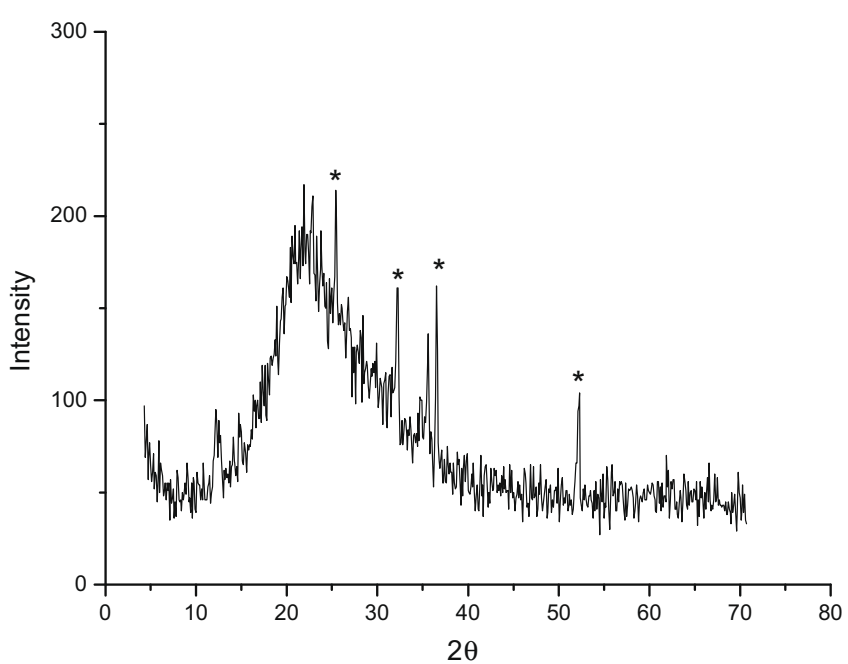

Fig. 8. XRD pattern for the $\mathrm{Co}-\mathrm{SiO}_{2} /$ sol-gel sample. ${ }^{*} \mathrm{Co}_{3} \mathrm{O}_{4}$.
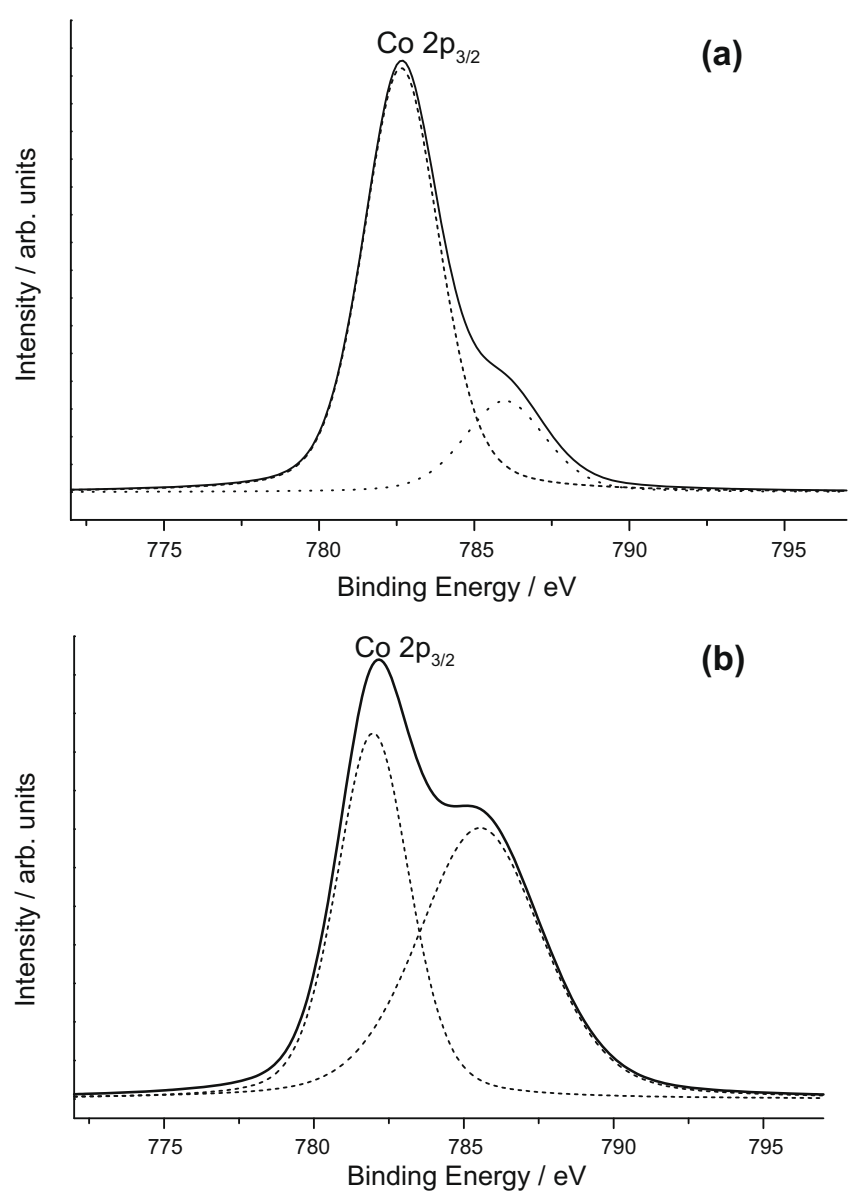

Fig. 9. Co $2 \mathrm{p}$ photoelectron spectra of the $\mathrm{Co}-\mathrm{SiO}_{2} /$ sol-gel (a) and $\mathrm{Co}-\mathrm{MCM}-41$ (b) samples.

components (ca. $782.5 \mathrm{eV}$ ) and intense satellite peaks at ca. $786 \mathrm{eV}$ indicate the presence of $\mathrm{Co}^{2+}$ ions on the surface of both catalysts. As the binding energy values exceed $782.0 \mathrm{eV}$, a strong interaction of the $\mathrm{Co}^{2+}$ ions with silica walls or even the incorporation of the $\mathrm{Co}^{2+}$ ions within the silica framework can be suggested [8,35]. A stronger shake-up satellite peak in the spectrum of Co-MCM-41 compared to that of $\mathrm{Co}-\mathrm{SiO}_{2} /$ sol-gel is indicative of the presence
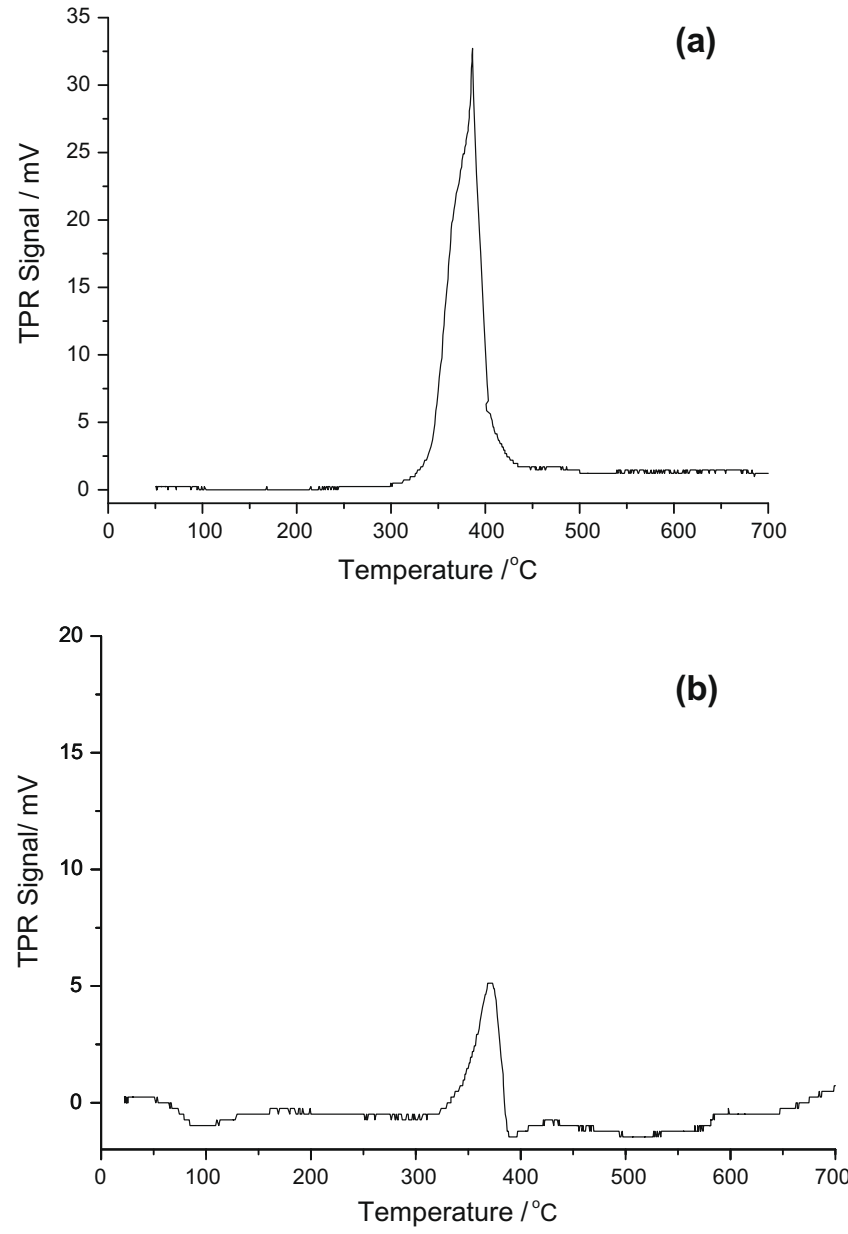

Fig. 10. TPR profiles of the $\mathrm{Co}-\mathrm{SiO}_{2} /$ sol-gel (a) and Co-MCM-41 (b) samples.

of $\mathrm{Co}^{2+}$ species in the octahedral symmetry as found in $\mathrm{CoO}$ [8]. Therefore, although XRD does detect the presence of small amounts of the $\mathrm{Co}_{3} \mathrm{O}_{4}$ phase in both samples (see above), their XP spectra exhibit the Co $2 \mathrm{p}_{3 / 2}$ peaks which could be assigned to the $\mathrm{Co}^{2+}$ ions in the isolated single-site state rather than to $\mathrm{Co}_{3} \mathrm{O}_{4}$ [36]. For $\mathrm{Co}_{3} \mathrm{O}_{4}$, a binding energy value of $780.0 \mathrm{eV}$ is expected [35], which is the intermediate value between those for $\mathrm{Co}^{3+}$ and $\mathrm{Co}^{2+}$. Thus, XPS results have shown that the introduced cobalt is present on the surface of both catalysts mostly as the isolated $\mathrm{Co}^{2+}$ ions.

The states of the cobalt species have been also investigated by $\mathrm{H}_{2}$-TPR (Fig. 10). The results obtained support the hypothesis that, at least in the Co-MCM-41 sample, cobalt seems to be essentially incorporated in the silica framework or attached to the surface via surface functional groups, e.g., hydroxyl groups. The TPR profile of $\mathrm{Co}-\mathrm{SiO}_{2} /$ sol-gel exhibits a broad low temperature peak at ca. $400{ }^{\circ} \mathrm{C}$ due to the consecutive reduction steps of the extraframework $\mathrm{Co}_{3} \mathrm{O}_{4}$ : a lower temperature reduction of $\mathrm{Co}^{3+}$ and $\mathrm{Co}^{2+}$, and a slightly higher temperature reduction of $\mathrm{Co}^{2+}$ and $\mathrm{Co}^{\circ}$. On the other hand, a low intensity of the corresponding peak in the TPR profile of $\mathrm{Co}-\mathrm{MCM}-41$ indicates that $\mathrm{Co}_{3} \mathrm{O}_{4}$ is present in this sample in small amounts. It should be also mentioned that in the TPR profiles of both samples there are no well-defined high temperature peaks (up to $700{ }^{\circ} \mathrm{C}$ ) suggesting a strong interaction of the $\mathrm{Co}^{2+}$ ions with silica matrices. This fact is expected to contribute valuably to the stability of the catalysts toward metal leaching under liquidphase oxidative conditions.

Thus, the characterization results show that in both samples cobalt is present on the surface mostly as the isolated $\mathrm{Co}^{2+}$ ions, with 
Table 2

Co-catalyzed oxidation of isolongifolene (1) under solvent-free conditions ${ }^{\mathrm{a}}$.

\begin{tabular}{|c|c|c|c|c|c|c|c|c|c|c|}
\hline \multirow[t]{3}{*}{ Run } & \multirow[t]{3}{*}{ Catalyst (wt\%) } & \multirow[t]{3}{*}{ Conversion (\%) } & \multicolumn{4}{|c|}{ Product selectivity (\%) } & \multirow[t]{3}{*}{$\mathrm{S}_{\text {allyl }}^{\mathrm{b}}(\%)$} & \multirow[t]{3}{*}{$\mathrm{S}_{\text {epox }}{ }^{\mathrm{b}}(\%)$} & \multirow[t]{3}{*}{$\mathrm{TON}^{\mathrm{c}}$} & \multirow[t]{3}{*}{$\operatorname{Rate}^{\mathrm{d}}\left(\mathrm{mmol} \mathrm{h}^{-1}\right)$} \\
\hline & & & \multicolumn{2}{|c|}{ Allylic oxidation } & \multicolumn{2}{|c|}{ Epoxidation } & & & & \\
\hline & & & 2 & 3 & 4 & 5 & & & & \\
\hline 1 & None & 0 & & & & & & & & \\
\hline 2 & $\mathrm{Co}-\mathrm{SiO}_{2} /$ sol-gel (2.8) & 46 & 60 & 13 & 9 & 3 & 73 & 12 & 74 & 0.46 \\
\hline 3 & $\mathrm{Co}-\mathrm{SiO}_{2} /$ sol-gel (4.2) & 80 & 60 & 7 & 2 & 9 & 67 & 11 & 85 & 0.70 \\
\hline 4 & Co-MCM-41 (2.8) & 48 & 84 & 7 & 6 & 3 & 88 & 9 & 122 & 0.48 \\
\hline 5 & Co-MCM-41 (4.2) & 85 & 85 & 5 & 6 & 2 & 90 & 8 & 138 & 0.73 \\
\hline $6^{e}$ & Co-MCM-41 (4.2) & 32 & 65 & 25 & 9 & - & 90 & 10 & 52 & 0.74 \\
\hline 7 & Co-MCM-41 (5.6) & 90 & 89 & 4 & 3 & 2 & 93 & 5 & 110 & 0.98 \\
\hline $8^{f}$ & Co-MCM-41 (2.8) & 7 & 58 & 34 & 7 & - & 92 & 7 & 11 & 0.49 \\
\hline $9^{g}$ & Co-MCM-41 (5.6) & 89 & 90 & 3 & 3 & 3 & 93 & 6 & 240 & 0.74 \\
\hline
\end{tabular}

a Conditions: $80^{\circ} \mathrm{C}, 1 \mathrm{~atm}\left(\mathrm{O}_{2}\right)$, reaction time $24 \mathrm{~h}$. Conversion and selectivity were determined by $\mathrm{GC}$.

b Selectivities for allylic oxidation and epoxidation products: $\mathbf{2}+\mathbf{3}$ and $\mathbf{4}+\mathbf{5}$, respectively. Ketone $\mathbf{5}$ is considered to be the product of the isomerization of epoxide $\mathbf{4}$ [16,18].

c TON - moles of the substrate converted/moles of Co.

d Initial rate of the conversion of isolongifolene.

e Reaction time $6 \mathrm{~h}$.

f The catalyst was removed by filtration after $2 \mathrm{~h}$ at the reaction temperature, and the filtrate was allowed to react further. The rate is given for the first $2 \mathrm{~h}$.

The catalyst was re-used after run 7; the total TON for two reaction cycles is given.

the amounts of the extraframework $\mathrm{CO}_{3} \mathrm{O}_{4}$ being higher in $\mathrm{Co}-\mathrm{SiO}_{2} /$ sol-gel than in Co-MCM-41. The latter can be understood taking into account the content of cobalt in the samples: $5.0 \mathrm{wt} \%$ in $\mathrm{Co}-$ $\mathrm{SiO}_{2} /$ sol-gel and $3.3 \mathrm{wt} \%$ in Co-MCM-41. In a previous work [10], the existence of a maximum value for the amounts of cobalt that can be incorporated in the MCM-41 framework was shown. For the synthetic method used in [10], this maximum was nearly $4 \mathrm{wt} \%$, after which a low temperature peak corresponding to the $\mathrm{Co}_{3} \mathrm{O}_{4}$ reduction appeared in the $\mathrm{H}_{2}$-TPR profiles of the samples.

\subsection{Catalytic studies}

The behavior of the prepared $\mathrm{Co}-\mathrm{SiO}_{2} /$ sol-gel and Co-MCM-41 samples was examined in the oxidation of isolongifolene (1) under non-solvent conditions. In all the experiments, these materials alone were applied as catalysts in neat liquid substrates under one atmosphere of molecular oxygen without the addition of bromide ions, which are usually used as auxiliary hydrogen abstraction agents in cobalt-catalyzed oxidation processes. The results are presented in Table 2.

Both cobalt-containing samples effectively catalyze the autoxidation of isolongifolene showing a similar activity, whereas in a blank reaction with no catalyst added no conversion has been observed at all (Table 2, run 1). The reaction gives only four major products (2-5) with a combined selectivity of $80-99 \%$ at $80-90 \%$ conversion, which is a rather high value for the reaction involving, more likely, free radicals. The distribution of the products depends on the reaction time; however, in all the runs compound $\mathbf{2}$ is a predominant product accounting for $60-90 \%$ of the total amounts of the products at the end of the reaction.

As the reaction occurs in neat substrate, high concentrations of valuable oxygenated products in final mixtures can be attained (up to $90 \mathrm{wt} \%$ ), which is one of the technological advantages of the method. Although several products are formed, for practical purposes their separation is often not necessary as the mixtures themselves show interesting organoleptic properties and can be used directly in fragrance compositions. The reaction becomes stagnated at high conversions. Depending on the catalyst amounts, the stagnation of conversion occurs at $50 \%$ to $90 \%$, afterward the catalyst has to be separated. The addition of the fresh catalyst to the reaction mixture after the stagnation promotes the additional conversion of the substrate. Moreover, the recovered catalyst after washing can be re-used several times without a significant loss of activity and selectivity (see below). Thus, it seems that the problem at high conversions is the accumulation of the products inside the pores of the catalyst which prejudices the access of the substrate molecules to the catalyst active sites.

It is important that the catalyst can be removed from the reaction mixture by simple centrifugation or filtration. Such a simple handling represents another attractive feature of the developed process.

The autoxidation of isolongifolene in the presence of $\mathrm{Co}-\mathrm{SiO}_{2} /$ sol-gel occurs smoothly at $80{ }^{\circ} \mathrm{C}$ resulting in a $46 \%$ conversion in $24 \mathrm{~h}$ (Table 2, run 2). Keeping the mixture under stirring for more time does not result in the further conversion of the substrate. On the other hand, with increased amounts of the catalyst, nearly $80 \%$ conversion has been obtained in $24 \mathrm{~h}$ (Table 2 , run 3 ). The results of these runs correspond to turnover numbers of 74 and 85 , respectively, with respect to the total amounts of cobalt in the material. However, the real efficiency of the surface cobalt species is much higher because most of the cobalt ions are obviously located in the bulk solid and are inaccessible for the substrate. In should be mentioned that the reaction rate is not affected by changes in the intensity of stirring, thus the diffusion limitations, if exist, seem to be intraparticle ones.

Four major products, all having the isolongifolane skeleton intact, have been identified in the reaction solutions in a combined selectivity of ca. $80 \%$ based on the reacted substrate, along with several minor unidentified products. Allylic oxidation products, i.e., isolongifolen-9-one (2) and isolongifolen-9-ol (3), account for ca. $70 \%$ of the mass balance, and epoxidation products, i.e., isolongifolene oxide (4) and isolongifolan-10-one (5) account for only ca. $10 \%$ (Schemes 1 and 2). Nearly $60 \%$ of the substrate has been converted into only one product, i.e., conjugated ketone $\mathbf{2}$. Saturated ketone $\mathbf{5}$ given in Table 2 is considered as an epoxidation product as it most likely results from the isomerization of epoxide $4[16,18]$.
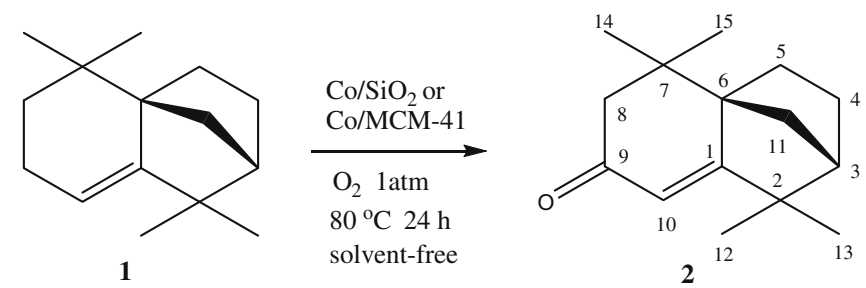

Scheme 1. Oxidation of isolongifolene. 


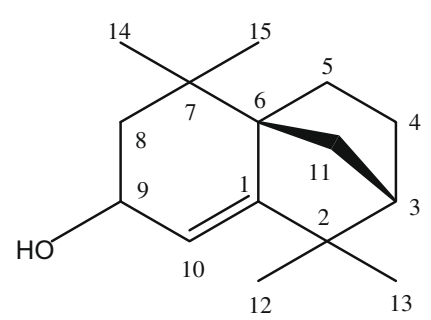

3

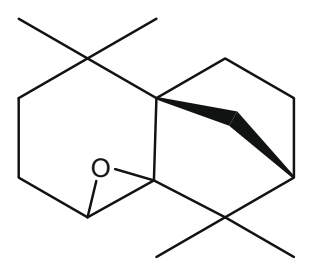

4

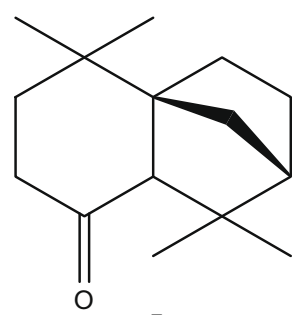

5

Scheme 2. Minor products of the oxidation of isolongifolene.

The Co-MCM-41 material also efficiently catalyzes the oxidation of isolongifolene (Table 2, runs 4 and 5 ). The product nature is similar to that obtained with $\mathrm{Co}-\mathrm{SiO}_{2} /$ sol-gel; however, the reaction selectivity is remarkably higher. The combined selectivity for products 2-5 reaches $98 \%$, in which ca. $85 \%$ is ketone 2 . The latter product seems to arise due to the further oxidation of primarily formed allylic alcohol $\mathbf{3}$ as the selectivity for $\mathbf{2}$ increases with the progress in the reaction at the expense of the alcohol $\mathbf{3}$ (Table 2 , cf. runs 5 and 6 ).

In spite of the surface area of Co-MCM-41 being four times higher than that of $\mathrm{Co}-\mathrm{SiO}_{2} /$ sol-gel and the cobalt content being only one-and-a-half times less (Table 1 ), their activities are quite similar (cf. runs 2 and 4; runs 3 and 5 in Table 2) demonstrating that the reaction might be complicated by mass transfer processes. On the other hand, the initial rate of the reaction is nearly proportional to the amounts of the catalyst for both $\mathrm{Co}-\mathrm{SiO}_{2} /$ sol-gel and Co-MCM-41 (Table 2). It seems, therefore, that in $\mathrm{Co}-\mathrm{SiO}_{2} /$ sol-gel a larger proportion of cobalt is accessible for the substrate due to the larger amounts of the extraframework $\mathrm{Co}_{3} \mathrm{O}_{4}$ or/and less amounts of small pores.

To control leaching of the active metal, the catalyst was filtered off at the reaction temperature after $2 \mathrm{~h}$ of using, and the filtrate was allowed to react further (Table 2, run 8). No additional conversion of isolongifolene was observed after the removal of the catalyst , which supported heterogeneous catalysis, i.e., the reaction solution contained no significant amounts of dissolved cobalt species and cobalt ions immobilized in the solid matrix were responsible for the oxidation of the substrate. In run 7 after the reaction, the catalyst was removed by centrifugation, washed with cyclohexane, and reused. The behavior of the spent catalyst with fresh substrate was nearly the same as that in the original reaction (Table 2, cf. runs 7 and 9). The total TON for two reaction cycles reached the value of 240 . Thus, the catalyst releases no cobalt into the medium, and can be easily recovered by either centrifugation or filtration and re-used. The total Co content in the spent catalyst after run 9 determined by ICP-AES was virtually the same as that in the fresh catalyst.

In the reactions of autoxidation of alkenes, a free radical chain mechanism is proposed which involves the formation of allylic hydroperoxides that typically decompose to several products [37]. The role of metal ions is generally explained in terms of catalysis of the decomposition of the allylic hydroperoxide intermediates which facilitates the initiation of the free radical chain mechanism. In cobalt-catalyzed homogeneous oxidations of alkenes, a competition between the abstraction of the allylic hydrogen to give allylic oxidation products and the addition of the alkylperoxy radical to the double bond resulting in epoxide products is usually expected [38]. In other words, allylic oxidation and epoxidation are competitive processes, and the contribution of each reaction strongly depends on the substrate nature and the relative stability of the corresponding intermediates [39]. A strong preference exhibited by isolongifolene for allylic oxidation over epoxidation can be explained by the high reactivity of the allylic hydrogen in this molecule toward the abstraction.
It seems that isolongifolene can adopt a conformation that allows posing the allylic hydrogen in the position that is nearly orthogonal to the double bond. In such an arrangement, the hydrogen abstraction is promoted by overlapping between the olefinic $\pi$-orbital and the developing p-orbital containing an unpaired electron in the transition state. Thus, the so-called "cyclic activation", which is an enhanced reactivity of cyclic allylic hydrogens compared to acyclic ones due to the initial arrangement of the molecule which is similar to that of the transition state in the course of the hydrogen abstraction [40], is successfully realized in the isolongifolene molecule. In our previous work, it has been observed that the cobalt-catalyzed oxidations of $\beta$-pinene and 3-carene also lead almost exclusively to allylic derivatives, while limonene and $\alpha$-pinene give both epoxides and allylic products [26]. It has been suggested that the allylic oxidation becomes a major reaction in the case of $\beta$-pinene and 3-carene because allylic radicals formed from these substrates have the structures that are favorable for the $\pi-p$ interaction. We suppose that a similar situation is realized in the case of isolongifolene. In addition, a steric congestion at the double bond in the isolongifolene molecule should also disfavor the epoxidation route.

The remarkably high selectivity of the liquid-phase oxidation of isolongifolene over the Co-MCM-41 catalyst deserves special attention. It is clear that a highly organized structure of this material exerts a pronounced shape-selective effect on the reaction. The $\mathrm{Co}-\mathrm{SiO}_{2} /$ sol-gel sample has larger pores and higher total pore volume than Co-MCM-41; however, it promotes a less selective reaction. Therefore, it seems that the shape of the pores is very important to maintain high selectivity during the reaction. The structure of a solid catalyst should allow the rapid diffusion of the bulky products throughout the porous medium to prevent successive reactions. In the case of Co-MCM-41, the highly ordered and uniform channel-like mesopores and their facile connectivity should favor the transport of the products away from the active sites. On the other hand, in $\mathrm{Co}-\mathrm{SiO}_{2} /$ sol-gel, the material with more heterogeneous pore shape distribution, ink-bottle pores with narrow mouths might be present so that intraparticle mass transport limitations could appear resulting in over-oxidation of the primarily formed products. In addition, due to the high surface uniformity of Co-MCM-41, the cobalt active sites are expected to be more energetically homogeneous than those in $\mathrm{Co}-\mathrm{SiO}_{2} / \mathrm{sol}-$ gel, which should also favor the selectivity. Taking into account that the reaction occurs in neat substrate, it is remarkable that the catalyst shows such a high selectivity even at the conversions of nearly $90 \%$. In other words, conjugated ketone $\mathbf{2}$ does not undergo significant further oxidation even being in close contact with the surface cobalt species under the applied conditions.

\section{Conclusions}

In summary, we have developed an efficient and environmentally friendly catalytic process for the oxidation of isolongifolene 
under mild aerobic conditions. The use of the inexpensive cobalt as the catalyst, molecular oxygen as the final oxidant, high reaction selectivity, and solvent-free conditions are significant practical advantages. The catalysts undergo no metal leaching, and can be easily recovered from the reaction mixture and re-used. The CoMCM-41 material has shown better performance than the sample prepared by the conventional sol-gel method without the surfactant. Further studies are targeted toward the applications of ordered metallosilicates for the oxidation of other renewable substrates.

\section{Acknowledgments}

The CNPq, CAPES, FAPEMIG, and INCT-Catálise (Brazil) are gratefully acknowledged for the financial support. The authors wish to thank the LNLS synchrotron laboratory, Campinas, Brazil, for permitting the use of the equipment, and Marcelo Rosmaninho for experimental assistance in the TPR measurements.

\section{References}

[1] I.W.C.E. Arends, R.A. Sheldon, Appl. Catal. A 212 (2001) 175

[2] R.A. Sheldon, R.S. Downing, Appl. Catal. A 189 (1999) 163.

[3] J.S. Rafelt, J.H. Clark, Catal. Today 57 (2000) 33.

[4] M. Ziolek, Catal. Today 90 (2004) 145

[5] C.T. Kresge, M.E. Leonowicz, W.J. Roth, J.C. Vartuli, J.S. Beck, Nature 359 (1992) 710.

[6] J.S. Beck, J.C. Vartuli, W.J. Roth, M.E. Leonowicz, C.T. Kresge, K.C. Chu, D.H Olson, E.W. Sheppard, S.B. McCullen, J.L. Schenkler, J. Am. Chem. Soc. 114 (1992) 10834.

[7] V. Parvulescu, B.-L. Su, Catal. Today 69 (2001) 315

[8] S.S. Bhoware, A.P. Singh, J. Mol. Catal. A 266 (2006) 118.

[9] T. Vrålstad, G. Øye, M. Stöcker, J. Sjöblom, Micropor. Mesopor. Mater. 104 (2007) 10.

[10] S. Lim, D. Ciuparu, S. Pak, F. Dobek, Y. Chen, D. Harding, L. Pfefferle, G. Haller, J. Phys. Chem. B 107 (2003) 11048.

[11] S. Lim, D. Ciuparu, Y. Yang, G. Du, L.D. Pfefferle, G.L. Haller, Micropor. Mesopor Mater. 101 (2007) 200.
[12] T. Somanathan, A. Pandurangan, D. Sathiyamoorthy, J. Mol. Catal. A 256 (2006) 193.

[13] B. Tyagi, M.K. Mishra, R.V. Jasra, Catal. Commun. 7 (2005) 52.

[14] B. Singh, J. Patial, P. Sharma, S.G. Agarwal, G.N. Qazi, S. Maity, J. Mol. Catal. A 266 (2007) 215.

[15] K.A. da Silva Rocha, P.A. Robles-Dutenhefner, I.V. Kozhevnikov, E.V. Gusevskaya, Appl. Catal. A 352 (2009) 188.

[16] E.J.Brunke, D. Schatkowski, US Patent 5,426,095, 1995.

[17] I. Bombarda, J. Smadja, J.Y. Conan, R. Faure, J. Agric. Food Chem. 42 (1994) 138.

[18] K.S. Ravikumar, J.-P. Bégué, D. Bonnet-Delpon, M. Ourévitch, J. Fluorine Chem. 102 (2000) 51.

[19] W. Pickenhagen, D. Schatkowski, US Patent 6,734,159, 2004.

[20] P.K. Srivastava, G. Chandra, Indian Perfumer 26 (1982) 151

[21] U.R. Nayak, V.S. Dalavoy, V.B. Deodhar, Indian J. Chem. B 28B (1989) 1055.

[22] A. Zhang, J. Carroll, S. Wang, J.A. Klun, US Patent 7,378,557, 2008.

[23] M.J. da Silva, P.A. Robles-Dutenhefner, L. Menini, E.V. Gusevskaya, J. Mol. Catal. A 201 (2003) 71

[24] P.A. Robles-Dutenhefner, M.J. da Silva, L.S. Sales, E.M.B. Sousa, E.V. Gusevskaya, J. Mol. Catal. A 217 (2004) 139.

[25] L. Menini, M.J. da Silva, M.F.F. Lelis, J.D. Fabris, R.M. Lago, E.V. Gusevskaya, Appl. Catal. A 269 (2004) 117.

[26] L. Menini, M.C. Pereira, L.A. Parreira, J.D. Fabris, E.V. Gusevskaya, J. Catal. 254 (2008) 355.

[27] M.J. da Silva, J.A. Gonçalves, O.W. Howarth, R.B. Alves, E.V. Gusevskaya, J. Organomet. Chem. 689 (2004) 302.

[28] M.G. Speziali, P.A. Robles-Dutenhefner, E.V. Gusevskaya, Organometallics 26 (2007) 4003.

[29] J.A. Gonçalves, E.V. Gusevskaya, Appl. Catal. A 258 (2004) 93.

[30] J.A. Gonçalves, M.J. da Silva, D. Piló-Veloso, O.W. Howarth, E.V. Gusevskaya, J. Organomet. Chem. 690 (2005) 2996.

[31] D. Helmig, F. Bocquet, J. Pollmann, T. Revermann, Atmos. Environ. 38 (2004) 557.

[32] S.J. Gregg, K.S.W. Sing, Adsorption, Surface Area and Porosity, Academic Press, London, UK, 1982.

[33] A. Corma, Chem. Rev. 97 (1997) 2373.

[34] M. Kruk, M. Jaroniec, Chem. Mater. 13 (2001) 3169.

[35] L. Guczi, D. Bazin, Appl. Catal. 188 (1999) 163.

[36] O. Tang, Q. Zhang, H. Wu, Y. Wang, J. Catal. 230 (2005) 384

[37] C.L. Bailey, R.S. Drago, Coord. Chem. Rev. 79 (1987) 321.

[38] R.A. Sheldon, J.K. Kochi, Metal-Catalyzed Oxidations of Organic Compounds, Academic Press, London, 1981. p. 133.

[39] E.F. Murphy, T. Mallat, A. Baiker, Catal. Today 57 (2000) 115.

[40] G. Rothenberg, Y. Yatziv, Y. Sasson, Tetrahedron 54 (1998) 593. 